\title{
Syntaxin-3 regulates newcomer insulin granule exocytosis and compound fusion in pancreatic beta cells
}

\author{
D. Zhu • E. Koo $・$ E. Kwan $\cdot$ Y. Kang $\cdot$ S. Park • \\ H. Xie $\cdot$ S. Sugita $\cdot$ H. Y. Gaisano
}

Received: 10 August 2012 / Accepted: 1 October 2012 / Published online: 7 November 2012

(C) Springer-Verlag Berlin Heidelberg 2012

\begin{abstract}
Aims/hypothesis The molecular basis of the exocytosis of secretory insulin-containing granules (SGs) during biphasic glucose-stimulated insulin secretion (GSIS) from pancreatic beta cells remains unclear. Syntaxin (SYN)-1A and SYN-4 have been shown to mediate insulin exocytosis. The insulinsecretory function of SYN-3, which is particularly abundant in SGs, is unclear.

Methods Mouse pancreatic islets and INS-1 cells were treated with adenovirus carrying Syn-3 (also known as Stx3) or small interfering RNA targeting Syn-3 in order to examine insulin secretion by radioimmunoassay. The localisation and distribution of insulin granules were examined by confocal and electron microscopy. Dynamic single-granule fusion events were assessed using total internal reflection fluorescence microscopy (TIRFM).

Results Depletion of endogenous SYN-3 inhibited insulin release. TIRFM showed no change in the number or fusion competence of previously docked SGs but, instead, a marked reduction in the recruitment of newcomer SGs and
\end{abstract}

D. Zhu and E. Koo contributed equally to this work.

Electronic supplementary material The online version of this article (doi:10.1007/s00125-012-2757-0) contains peer-reviewed but unedited supplementary material, which is available to authorised users.

D. Zhu • E. Koo • E. Kwan • Y. Kang • H. Xie • H. Y. Gaisano $(\bowtie)$ Department of Medicine, University of Toronto,

Toronto, ON, Canada M5S 1A8

e-mail: herbert.gaisano@utoronto.ca

S. Park $\cdot$ S. Sugita $\cdot$ H. Y. Gaisano

Department of Physiology, University of Toronto,

Toronto, ON, Canada

S. Park $\cdot$ S. Sugita

Division of Fundamental Neurobiology,

University Health Network,

Toronto, ON, Canada their subsequent exocytotic fusion during biphasic GSIS. Conversely, overexpression of Syn-3 enhanced both phases of GSIS, owing to the increase in newcomer SGs and, remarkably, to increased SG-SG fusion, which was confirmed by electron microscopy.

Conclusions/interpretation In insulin secretion, SYN-3 plays a role in the mediation of newcomer SG exocytosis and SG-SG fusion that contributes to biphasic GSIS.

Keywords Exocytosis · Granule-granule fusion · Newcomer insulin granules $\cdot$ Syntaxin-3 $\cdot$ TIRFM

$\begin{array}{ll}\text { Abbreviations } \\ \text { Ad } & \text { Adenovirus } \\ \text { EGFP } & \text { Enhanced green fluorescent protein } \\ \text { EM } & \text { Electron microscopy } \\ \text { FWHM } & \text { Full width at half maximum } \\ \text { GLP-1 } & \text { Glucagon-like peptide-1 } \\ \text { GSIS } & \text { Glucose-stimulated insulin secretion } \\ \text { IAPP } & \text { Islet amyloid polypeptide } \\ \text { IBMX } & \text { 3-Isobutyl-1-methylxanthine } \\ \text { Munc18b } & \text { Mammalian homologue of unc-18b } \\ \text { NPY } & \text { Neuropeptide Y } \\ \text { PM } & \text { Plasma membrane } \\ \text { RNAi } & \text { RNA interference } \\ \text { SG } & \text { Secretory insulin-containing granule } \\ \text { Si } & \text { Small interfering } \\ \text { SM } & \text { Sec1/Munc18-like protein } \\ \text { SNAP25/23 } & \text { Synaptosomal-associated protein of } \\ & \text { 25/23 kD } \\ \text { SNARE } & \text { Soluble } N \text {-ethylmaleimide-sensitive fusion } \\ \text { SYN } & \text { protein-attachment protein receptor } \\ \text { t- } & \text { Syntaxin } \\ \text { TIRFM } & \text { Target- } \\ & \text { Total internal reflection fluorescence } \\ & \text { microscopy }\end{array}$




$\begin{array}{ll}\text { v- } & \text { Vesicle- } \\ \text { VAMP } & \text { Vesicle-associated membrane protein } \\ \text { WT } & \text { Wild type }\end{array}$

\section{Introduction}

Glucose-stimulated insulin secretion (GSIS) from pancreatic islet beta cells displays a biphasic pattern both in vivo and in vitro [1]. The exocytosis of secretory insulin-containing granules (SGs) that underlies each of the two phases of insulin secretion may differ spatially and involve distinct membrane-fusion machineries. Soluble $N$-ethylmaleimidesensitive fusion protein-attachment protein receptor (SNARE) proteins, including vesicle-associated membrane protein (VAMP), synaptosomal-associated protein of $25 \mathrm{kD}$ (SNAP25) and syntaxin (SYN), are the fundamental components of the exocytotic machinery required for docking and fusion of SGs with plasma membrane (PM) in neurons [2]. These SNARE proteins are produced in pancreatic beta cells and mediate similar putative steps in SG exocytosis [3]. Each vesicle (v)- and target ( $\mathrm{t}$ )-membrane SNARE protein constitutes a large family of isoforms [4]. In beta cells, combinatorial matching of $\mathrm{v}$ - and t-SNARE isoforms may form the molecular basis of the distinct exocytotic events underlying the two phases of GSIS. It is suggested that, in addition to docked SGs that are purported to form the readily releasable pool (RRP) that accounts for firstphase insulin release, large numbers of SGs, designated 'newcomer SGs', are mobilised from the cell interior to contribute not only to second-phase but also to first-phase release [5-7]. Insulin exocytosis also involves compound exocytosis of SGs when potentiated by cAMP-acting glucagon-like peptide 1 (GLP-1) [8] or $\mathrm{Ca}^{2+}$-acting carbachol [9].

Of the four syntaxins mediating exocytosis, SYN-1, SYN-2 and SYN-4 are present and localised predominantly to the beta cell PM, whereas SYN-3 is noted to be more abundant in SGs [10]. Pancreatic islets from Syn-1a (also known as Stxla)-knockout mice exhibited blunted firstphase secretion, and total internal reflection fluorescence microscopy (TIRFM) of single beta cells demonstrated that this was due to loss of ability of previously docked SGs to undergo exocytosis, but without perturbation of the recruitment and fusion of newcomer SGs [11]. SYN-4 has been suggested to influence insulin secretion, predominantly first-phase release, but the precise exocytotic step at the level of single SG fusion remains unknown [12]. The roles of SYN-2 and SYN-3 in insulin secretion are unknown. As SYN-3 appears to be the only SYN localised to SGs, we can surmise that SYN-3 possibly influences newcomer SG fusion and/or SG-SG fusion $[3,8,13]$. We thus proceeded to examine this hypothesis.
Numerous studies on distinct cell types have demonstrated the cell-context-specific role of SYN-3 in regulating secretion. In kidney cells, SYN-3 was implicated in apical membrane docking and fusion [14]. In gastric glands, SYN3 regulated parietal acid secretion in complex with mammalian homologue of unc-18b (Munc18b) and SNAP25 [15]. In retinal cells, SYN-3 is produced in ribbon synapses but its role in neurotransmitter release remains unknown $[16,17]$. In pancreatic acinar cells [18], SYN-3 was localised to zymogen granule membrane [19] and complexed with VAMP8 to mediate SG-SG fusion [20].

In the present study, we found that depletion of endogenous SYN-3 by RNA interference (RNAi) inhibited GSIS in insulinoma INS-1 cells. TIRFM showed that SYN-3-depleted INS-1 cells displayed severely impaired recruitment and fusion of newcomer SGs in both the first and second phases of GSIS, whereas fusion of previously docked SGs was unperturbed. Overexpression of Syn-3 (also known as Stx3) enhanced biphasic GSIS, an action that could be directly attributed to increased exocytosis of newcomer SGs. Remarkably though, Syn-3 overexpression also enhanced SG-SG fusion. Thus, we propose that SYN-3 in SGs facilitates mobilisation of newcomer SGs to the PM and SG-SG fusion, and that these two exocytotic events contribute to both the first and second phases of GSIS in beta cells.

\section{Methods}

Cell culture, Syn-3 small interfering RNA and Syn-3 adenovirus transduction INS-1 cells were cultured in RPMI $1640 \mathrm{Me}-$ dium (Gibco, Langley, OK, USA) at $37^{\circ} \mathrm{C}$ in $5 \%$ (vol./vol.) $\mathrm{CO}_{2}$. Syn-3 RNAi duplex (Dharmacon, Chicago, IL, USA) was transfected into INS-1 cells by Lipofectamine RNAiMAX (Invitrogen, Burlington, ON, Canada). The nontargeting pool of small interfering (si)RNAs was used in control cells. Syn-3 adenovirus was generated and transduced into islets or cells as indicated. For further details, please refer to the electronic supplementary material (ESM Methods). Islets were isolated from 3-6-month-old mice and beta-cells were dispersed as described more fully in the ESM Methods

Western blotting Western blots of islet lysate samples were performed with antibodies to the following: SYN-1A, SYN-2, SYN-3, SYN-4, synaptosomal-associated protein of $23 \mathrm{kD}$ (SNAP23), SNAP25, Munc18a, Munc18b, Munc18c, VAMP2, VAMP8 and beta actin. Protein bands were visualised by chemiluminescence (Pierce, Nepean, ON, Canada). For further details, please refer to the ESM Methods.

Insulin secretion Perifusion assays on mouse pancreatic islet and static insulin secretion from INS-1 cells were 
performed as reported previously [21], with secreted insulin determined by RIA (Linco, St Louis, MO, USA). Results are presented as insulin secreted normalised to total insulin content. For further details, please refer to the ESM Methods.

Confocal microscopy Samples were fixed and immunostained as described in the ESM Methods. Images were examined using a LSM510 laser scanning confocal imaging system. Please refer to the ESM Methods for further details.

Electron microscopy Islets were fixed and sections were examined and photographed with a Hitachi H-7000 transmission electron microscopy (EM) system as described previously [8]. Please refer to the ESM Methods for further details.

TIRFM and data analysis TIRFM images were acquired using a Nikon TE2000U TIRF microscope at $5 \mathrm{~Hz}$ with $100 \mathrm{~ms}$ exposure time. Fusion events, observed as flashes of fluorescence indicating emptying of the neuropeptide $\mathrm{Y}$ (NPY)-enhanced green fluorescent protein (EGFP) cargo, were manually selected, as recently reported in detail [22] (and see ESM Methods). Briefly, an increase of EGFP fluorescence exceeding $\times 5$ the standard deviation of basal fluorescence fluctuation was considered to represent a fusion event. Two concentric circles ( 5 and 7 pixels with pixel size of $267 \mathrm{~nm}$, corresponding to approximately 1.3 and $1.8 \mu \mathrm{m}$ diameters) were used to centre on selected SGs and characterise the change in fluorescence over time for a single SG. The dissipation of average fluorescence in the concentric annulus was taken as the reporter of release of granule cargo. Fusion events were also indicated by abrupt brightening of pHluorin fluorescence, and were manually selected for analyses of fusion-pore lifetime and compound exocytosis. The time period between onset of the pHluorin fluorescence brightening and peak fluorescence was defined as the fusion-pore lifetime. Mobilisation and exocytosis of SGs were analysed by Matlab (Math-Works, Natick, MA, USA), ImageJ (NIH, Bethesda, MD, USA) and Igor Pro software (WaveMetrics, Portland, OR, USA), as described in detail in the ESM Methods.

Statistical analysis All data are presented as means \pm SEMs. Statistical significance was evaluated by Student's $t$ test, repeated-measure ANOVA and Mann-Whitney rank sum test using SigmaStat (Systat Software, Chicago, IL, USA). Significant differences are indicated by asterisks $\left({ }^{*} p<0.05,{ }^{* * *} p<0.01\right)$.

\section{Results}

SYN-3 is a $t$-SNARE on SGs We examined the endogenous level of SYN-3 in mouse islet beta cells, and found SYN-3 to exhibit a punctate fluorescence appearance that colocalised with SGs (Fig. 1a). This was also the case with INS-1 cells, in which SYN-3-GFP fluorescence co-localised with SGs (Fig. 1b). By recording the movement of SYN-3GFP and SGs under TIRFM, we found that both green and red fluorescent spots displayed coincident mobility kinetics (Fig. 1c, d). These results demonstrate that SYN-3 is abundant on SGs in pancreatic beta cells and insulinoma cell lines.

Knockdown of SYN-3 diminishes biphasic GSIS by decreasing newcomer $S G$ fusion To investigate the endogenous function of SYN-3 in insulin secretion, we employed RNAi to acutely downregulate SYN-3 levels. Western blots confirmed that endogenous SYN-3 levels were depleted by $70.6 \%$ (Fig. 2a, b), whereas levels of Munc18a, $-18 \mathrm{~b}$ and $-18 \mathrm{c}$, SYN-1, -2 and -4 , SNAP23, SNAP25, VAMP2 and VAMP8 were not altered by SYN-3 depletion (Fig. 2a). Reduction of endogenous SYN-3 levels impaired GSIS by $42 \%(5.1 \pm 0.4 \%$ of insulin content) compared with control $(8.7 \pm 0.3 \%$ of insulin content) (Fig. 2c), but had no effect on basal secretion $(0.8 \mathrm{mmol} / 1$ glucose) or cellular insulin content (control, $1.12 \pm 0.04 \mu \mathrm{g} / \mathrm{well}$; with SYN-3 knockdown, $1.10 \pm$ $0.05 \mu \mathrm{g} / \mathrm{well})$.

We explored how changes in the spatial distribution of SGs as examined by EM might partly explain the above results. At resting or basal conditions, there were no differences between control and SYN-3 knockdown cells in total number of SGs, the number of SGs that were morphologically docked ( $<50 \mathrm{~nm}$ from PM [23]), or number of SGs close to the PM (within $120 \mathrm{~nm}$ ). However, after stimulation with $11.1 \mathrm{mmol} / 1$ glucose plus $10 \mathrm{nmol} / \mathrm{l} \mathrm{GLP}-1$ to greatly potentiate the recruitment of SGs to the PM [8], the number of docked SGs (Fig. 2d, e) and SGs close to the PM (ESM Fig. 1a) was reduced by $45.5 \%$ and $37.6 \%$, respectively, in Syn-3-silenced INS-1 cells compared with control cells, whereas the total number of SGs did not change (ESM Fig. 1b). This suggests that after docked SGs had undergone exocytosis, there was subsequent impairment of SGs to mobilise to the PM to replenish this stimulation-induced depletion of docked SGs in SYN-3-knockdown cells. EM, however, could only provide snapshots of SG behaviour, and could not be used to assess the dynamics of SG mobilisation and exocytotic fusion.

In order to visualise the spatio-temporal mobilisation of populations of SGs and single SG fusion dynamics, we employed time-lapse TIRFM to monitor exocytosis of SGs tagged with NPY-EGFP by adenovirus transduction (Ad$N p y$-EGFP) in INS-1 cells. At the basal state (Fig. 3a, b), levels of punctuate fluorescence indicating docked SGs were not different between control $(1.05 \pm 0.058$ per $\left.10 \mu^{2}\right)$ and SYN-3-knockdown INS cells $(1.03 \pm 0.068$ per $10 \mu \mathrm{m}^{2}$ ). However, when maximally stimulated by 

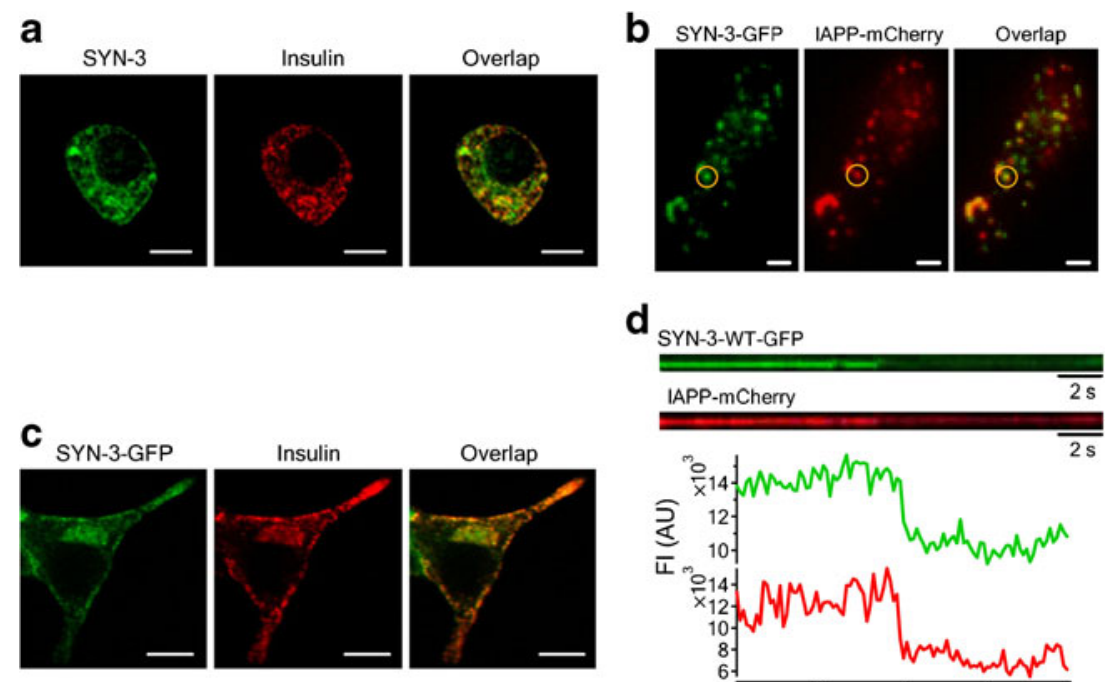

$\mathbf{d}_{\mathrm{s}+{ }_{3}}$

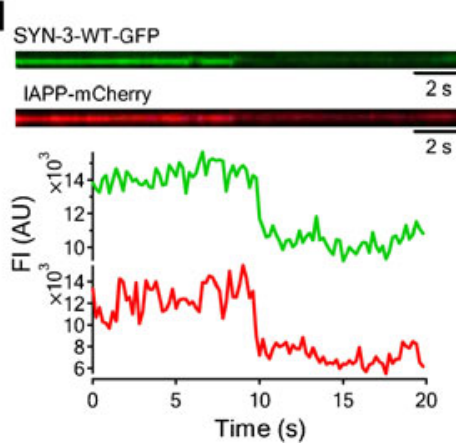

Fig. 1 SYN-3 is located on insulin granules. (a, b) Immunofluorescence images of SYN-3 (green), insulin (red) and their co-localisation, as shown in merged images (yellow), in (a) mouse pancreatic islet beta cells and (b) INS-1 cells. Scale bars, $5 \mu \mathrm{m}$. (c) An example showing partial co-localisation of SYN-3-WT and SGs (labelled by islet amyloid polypeptide [IAPP]-mCherry) in INS-1 cells under TIRFM. Scale

$16.7 \mathrm{mmol} / \mathrm{l}$ glucose plus $10 \mathrm{nmol} / \mathrm{l} \mathrm{GLP}-1$ and $150 \mu \mathrm{mol} /$ 13 -isobutyl-1-methylxanthine (IBMX) (GLP-1 plus IBMX maximally raises cAMP levels), SYN-3-knockdown cells displayed lower insulin-secretory capability compared with control cells (Fig. 3c). On stimulation, single SG fusion events were observed as flashes of fluorescence that rapidly dissipated in a cloud-like diffusion pattern. These exocytotic events were, however, not uniform but could be categorised into three distinct modes of exocytosis (Fig. 3d). 'Pre-dock' fusion mode (Fig. 3d top; blue in Fig. 3e, f) refers to SGs that were already docked onto PM (Fig. 3a) for a period of time prior to stimulation. 'Newcomer SGs' were new SGs appearing de novo under evanescent field after stimulation and which then underwent exocytosis. Newcomer SGs could be further categorised into two distinct patterns as described previously $[5,7,24]$, designated as 'no-dock' (middle, Fig. 3d) and 'short-dock' (bottom, Fig. 3d) newcomers. 'No-dock' newcomer SGs (red in Fig. 2e, f) were newly recruited by stimulation and immediately fused with the PM (docking state of $<200 \mathrm{~ms}$, the minimal interval between two consecutive frames). 'Short-dock' newcomer SGs (green in Fig. 2e, f) were those newly recruited by stimulation, which first docked for some time - varying from seconds to minutes - at the PM, and then fused with the PM.

We assessed the pattern(s) of fusion events impacted by knockdown of SYN-3 in INS-1 cells. At $2.8 \mathrm{mmol} / 1$ glucose in the presence of GLP-1, there were few spontaneous fusion events of mainly pre-dock SGs (Fig. 3e, f, g). With high-glucose stimulation, fusion events from pre-dock SGs bars, $2 \mu \mathrm{m}$. (d) Kymographs and corresponding time-lapse fluorescence-intensity curves (green, SYN-3-GFP; red, IAPPmCherry) from the SG indicated by white circles in (c). Data shown are representative of three or four independent experiments for each; $\mathrm{AU}$, arbitrary units

(Fig. 3h, i) were similar between control and SYN-3knockdown cells in the first phase (first 5 min after glucose stimulation), and also the second phase (from 5 to $18 \mathrm{~min}$ after glucose stimulation) of secretion. Remarkably, reduction of exocytosis in both phases of secretion could be entirely accounted for by the reduction in newcomer SG exocytosis. Specifically, in the first phase of GSIS, there was inhibition of both no-dock (red bars in Fig. 3e and f; control $6.50 \pm 0.95$ vs SYN-3 knockdown $1.88 \pm 0.52$ events/ $100 \mu \mathrm{m}^{2}$, same unit is used below) and short-dock (green bars in Fig. 3e and f; control $4.47 \pm 0.81$ vs SYN-3 knockdown $2.05 \pm 0.33$ ) newcomer SGs (Fig. 3h). In the second phase of GSIS, there was significant reduction in only nodock newcomer SGs (control 5.42 \pm 1.26 vs SYN-3 knock-

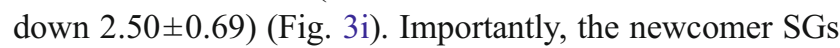
already accounted for $>80 \%$ of exocytotic events in firstphase GSIS in control cells (Fig. 3e). Thus, SYN-3 seemed to account for a larger portion of first-phase GSIS by mediating the recruitment and fusion of newcomer SGs, even though SYN-3 had no effects on previously docked SGs, the latter being attributed to SYN-1A [11].

To elucidate how SYN-3 might be involved in SG exocytotic fusion per se, we rigorously analysed the SG docking and fusion dynamics. We captured images, starting from when the SG was not yet visible and continuing to when the fluorescence of SG appeared and progressed to a nearly constant fluorescence. Some SGs kept stationary in the lateral plane with a relatively stable fluorescence, which we defined as the docking step preceding fusion with the 

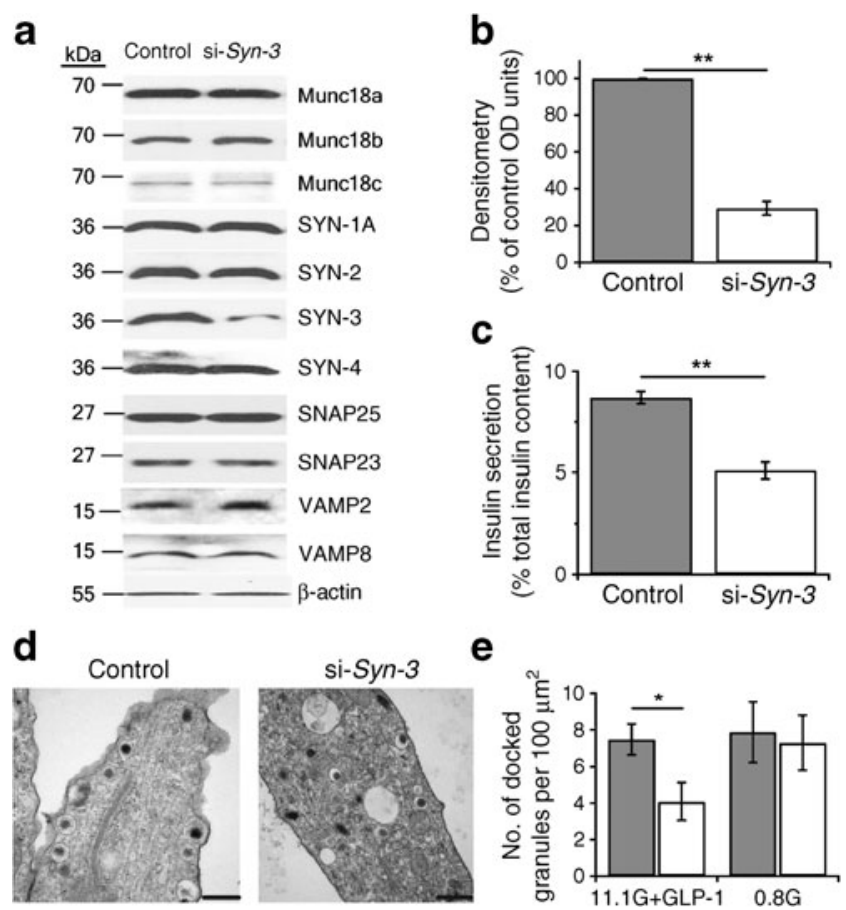

Fig. 2 Depletion of SYN-3 impairs GSIS. (a) Western blotting to verify RNAi-mediated acute knockdown of SYN-3 levels in INS-1 cells, and levels of SM and SNARE proteins as indicated. Data shown are representative of three independent experiments. (b) Densitometry analysis of SYN-3 levels from three experiments using RNAi-mediated SYN-3knockdown in INS-1 and control cells. (c) GSIS with $10 \mathrm{mmol} / 1$ glucose. Data represent the means \pm SEMs of three independent experiments; ${ }^{* *} p<0.01$. (d) Representative EM images after 3 min stimulation with $11.1 \mathrm{mmol} / \mathrm{l}$ glucose plus $10 \mathrm{nmol} / \mathrm{l}$ GLP-1 from control and SYN-3knockdown INS-1 cells. (e) Histograms of the number of SGs docked onto the PM before and after stimulation, as indicated from control (grey bars) and SYN-3-knockdown (white bars) INS-1 cells. $n=15-19$ per group. Summary graphs represent the means \pm SEMs. ${ }^{*} p<0.05 ; 0.8 \mathrm{G}$, $0.8 \mathrm{mmol} / 1$ glucose; $11.1 \mathrm{G}, 11.1 \mathrm{mmol} / \mathrm{l}$ glucose; No., number; si-Syn3, SYN-3-knockdown cells

PM; the time an SG stayed in this state prior to fusion we calculated as the docking dwell time (an example profile of a short-dock newcomer SG is shown in Fig. 3d). There was no difference in docking dwell times between control and SYN-3 knockdown cells (Fig. 3j-1).

Sometimes, however, we observed in SYN-3 knockdown cells that the EGFP fluorescence of SGs underwent a slower increase to reach peak fluorescence. With EGFP, it was difficult to determine whether the slower increase in fluorescence was due to the slow approach of SGs to the PM, or to the slow release of cargo from exocytosing SGs. To critically assess this process, we labelled SGs with NPY tagged with the $\mathrm{pH}$-sensitive GFP variant $\mathrm{pHluorin}$. In these conditions, SGs would be almost invisible when docked onto the PM, and would only start to fluoresce on opening of the SG fusion pore, at which time the SG lumen would become exposed to the alkaline $\mathrm{pH}$ of the cell exterior and the pHluorin would fluoresce $[25,26]$. We defined the period from the starting point of an SG beginning to fluoresce to then reach peak fluorescence as the fusion-pore lifetime. In control cells, SGs undergoing exocytosis exhibited fast fusionpore lifetimes, with a mean time constant of $<2 \mathrm{~s}$ (Fig. 3d, m), similar to that reported by Barg et al [27]. In SYN-3knockdown cells, exocytotic SGs exhibited a much slower increase in fluorescence, taking several seconds to tens of seconds before reaching peak fluorescence (Fig. 3n). Whereas no SGs displayed slow fusion-pore lifetimes in control cells, in SYN-3-knockdown cells, $70 \%$ of cells were found with fusion events displaying slow fusion-pore lifetimes (Fig. 3o); these accounted for $16.7 \%$ of the total fusion events per cell (Fig. 3p). This feature of slow fusion-pore lifetime was almost entirely observed in the population of no-dock newcomer SGs. These results led us to speculate that the dominant role of SYN-3 might not be in the docking step of newcomer SGs per se but, rather, in the priming and final fusion steps.

Gain of function of SYN-3 increases recruitment and fusion of newcomer SGs for enhanced biphasic GSIS The above studies focused on loss of function. Gain of function is of importance in rescuing deficient biphasic GSIS in diabetes $[3,28]$. We thus examined what would happen if we overexpressed Syn-3 to cause gain of function. We transduced INS-1 cells with adenovirus encoding Syn-3 (Ad-Syn-3GFP). Western blotting verified the increase in SYN-3 in INS-1 cells, along with Ad-Syn-1a-wild-type (WT) and its gain-of-function mutant Ad-Syn-1a-open form (OF; L165A/ E166A mutant) [29] (Fig. 4a). Syn-3 overexpression (over $90 \%$ transfection efficiency by GFP fluorescence) resulted in GSIS enhanced by $42.8 \%$ (9.6 $\pm 0.15 \%$ of insulin content $)$ compared with control $(6.5 \pm 0.94 \%$ of insulin content). The secretion potentiated by Syn-3 overexpression was comparable with that induced by $S y n-1 A-\mathrm{OF}$, while basal insulin secretion among all groups remained unchanged (Fig. 4b).

We determined which phase(s) of GSIS would be affected by the increased levels of islet SYN-3 by performing perifusion assays on pancreatic islets transduced with AdSyn-3-GFP-WT. Overexpression assessed by western blotting showed SYN-3-GFP levels were 2.3-fold those of endogenous SYN-3 (Fig. 4c). Serial Z-scanning by confocal microscopy showed GFP fluorescence reaching the islet core (ESM Fig. 2) but leaving some cells not transduced, suggesting that the increased levels of SYN-3 in the single beta cells examined would be even higher. Perifusion analysis of Ad-Syn-3-GFP-WT transduced islets displayed a dramatic increase in both first $(6.57 \pm 0.75)$ and second phases $(4.67 \pm 0.68)$ of GSIS compared with control (AdGFP, $3.09 \pm 0.42$ and $2.15 \pm 0.12$, respectively), such that total GSIS in Ad-Syn-3-GFP-WT-transduced islets was $215 \%$ that of control islets (Fig. 4d, analysis in Fig. 4e). Basal insulin release between the two groups was similar, as was total cellular insulin content. 
We next determined whether these exocytotic events might be affected by Syn-3 overexpression. INS-1 cells were transduced with Ad-Syn-3-mCherry. Transduced INS-1 cells exhibited markedly enhanced GLP-1-potentiated GSIS (Fig. 4f, g). We assessed the exocytotic events by TIRFM as in Fig. 3. Syn-3-overexpressing cells showed that the number of no-dock newcomer SGs undergoing exocytosis increased by $98 \%$ and $164 \%$ in first $(17.13 \pm 3.62$ vs control $8.66 \pm 1.19)$ and second phases $(24.60 \pm 5.92$ vs control 9.32 \pm 2.03 ), respectively. Short-dock newcomer SGs in Syn-3overexpressing cells were increased, but mainly in the first phase $(6.13 \pm 0.61$ vs control $2.45 \pm 0.45)$ (Fig. $4 \mathrm{f}-\mathrm{i}$ ).

Role of $S Y N-3$ in $S G-S G$ fusion As SG-SG fusion contributes to GLP-1-potentiated GSIS [8], and SYN-3 co-localises with SGs (Fig. 1), we postulated that SYN-3 could be a putative t-SNARE for homotypic SG-SG fusion [28]. To address this, we performed EM of Syn-3-overexpressing pancreatic islets and control islets stimulated by $11.1 \mathrm{mmol} / \mathrm{l} \mathrm{glu}-$ cose plus GLP-1, a condition that greatly induces SG-SG fusion [8]. Remarkably, SG-SG fusion events were much more abundant in Syn-3-overexpressing islet beta cells $\left(19.50 \pm 3.24 / 100 \mu \mathrm{m}^{2}\right)$ than in control beta cells $(9.27 \pm 1.45 /$ $100 \mu^{2}$ ) (Fig. 5a, b). The total number of SGs, SGs close to the PM (within $120 \mathrm{~nm}$ ) and SGs morphologically docked on PM were comparable between the two groups (ESM Fig. 3). Sequential SG-SG fusion and compound exocytosis have been studied in recent years, in various cell types, with multi-photon imaging $[30,31]$ and other imaging modalities [9]. Realising that static EM images could not distinguish these exocytotic events, we employed TIRFM to rigorously and quantitatively assess the spatio-temporal dynamics of these two types of SG-SG fusion: sequential SG-SG fusion and true compound fusion. We captured discrete fusion events of SGs labelled with syncollin-pHluorin [32] triggered by $16.7 \mathrm{mmol} / 1$ glucose and GLP-1 stimulation. Syncollin, a native cargo protein of pancreatic acinar cells, could reliably be targeted to SGs [33]. In addition to typical single flashes indicating exocytosis of single SGs, we observed other types of fusion event. Of note, in $14 \%$ of total exocytotic events in control cells, there was another flash occurring during the decay phase of the first fusion event (Fig. 5c), and sometimes we observed at precisely the same site a sequence of double or triple flashes following initial fusion events, indicating longer chains of SG-SG fusions. These sequential fusion events are consistent with the 'sequential exocytosis' described previously [31]. In 3\% of total fusion events, the fluorescence increase that occurred was not always associated with well-resolved sequential steps of similarly sized single SG flashes, but rather displayed massive fluorescence spots. An example of massive fusion is shown in Fig. 5d (analysed in the graph), and is further analysed in Fig. $5 \mathrm{f}$ left image, and compared with a
Fig. 3 Depletion of SYN-3 inhibits exocytosis of newcomer insulin granules and slows down exocytotic fusion. (a) TIRFM images of docked SGs from control or SYN-3-knockdown INS-1 cells. Scale bars, $2 \mu \mathrm{m}$. (b) Comparison of averaged docked SG densities before stimulation. (c) Normalised cumulative fusion events of SGs per $100 \mu \mathrm{m}^{2}$ from control (black circles) or SYN-3-knockdown (white circles) cells stimulated as indicated. (d) Kymographs and corresponding fluorescence-intensity curves showing three modes of SG fusion in INS-1 cells. Colour coding for the three kymographs in e and f: pre-dock, blue bar; newcomer-no dock, red bar; newcomer-short dock, green bar. (e, f) Histogram of the different fusion events in first phase (first $5 \mathrm{~min}$ after $16.7 \mathrm{mmol} / \mathrm{l}$ glucose stimulation) and second phase (5-18 min) of GSIS from control (e) or SYN-3-knockdown (f) cells. Data were obtained from six independent experiments $(n=2-4$ cells for each experiment), expressed as means \pm SEMs. (g-i) Summary of three modes of fusion event in $2.8 \mathrm{mmol} / \mathrm{l}$ glucose (g), first phase (h) and second phase (i) of $16.7 \mathrm{mmol} / 1$ glucose stimulation, shown as means \pm SEMs. Grey bars, control; white bars, SYN-3-knockdown cells. ${ }^{*} p<0.05 ;{ }^{* *} p<0.01 ;{ }^{* * *} p<0.001$. (j-l) Histograms $(\mathbf{j}, \mathbf{k})$ and cumulative distribution (l) of SG docking dwell times are shown for control $(\tau=68.7 \mathrm{~s}$ in [j]; black circles in [1]) and SYN-3-knockdown $(\tau=60.8 \mathrm{~s}$ in [k]; white circles in [1]) INS-1 cells. $n=10$ cells per group. $(\mathbf{m}, \mathbf{n})$ Representative examples showing the fast fusion-pore lifetime kinetics (m) of an SG in a control cell and slow fusion-pore lifetime kinetics (n) in a SYN-3-knockdown cell. (o) Comparison of the proportion of cells with slow fusion-pore lifetimes between control and SYN-3knockdown INS-1 cells. White bar, fast fusion-pore lifetime; black bar, slow fusion-pore lifetime. (p) Ratio of fusion events per cell with slow fusion-pore lifetimes. $n=15$ cells per group, shown as means \pm SEMs. ${ }^{* * *} p<0.001 .2 .8 \mathrm{G}, 2.8 \mathrm{mmol} / 1$ glucose; $16.7 \mathrm{G}, 16.7 \mathrm{mmol} / 1$ glucose; FI, fluorescence intensity; FP, fusion pore; No., number; si-Syn-3, SYN3-knockdown cells

typical single SG fusion event shown in the corresponding image and graphical analysis in Fig. 5e, f. In Fig. 5f, the graphical analysis of full width at half maximum (FWHM) (indicated by red arrows) shows these massive fusion events (mean $0.476 \mu \mathrm{m}$ ) to be twofold larger than single SG events (mean $0.242 \mu \mathrm{m}$ ). These massive fusion events could be interpreted as either swelling of SGs or exocytosis of several compound SGs. Our data support that latter for the following reasons. First, syncollin labels SG cargo. There is no accumulation of fluorescence caused by extracellular fluorescent probes or dyes refilling during fusion-pore opening, as is the case with post-fusion labelling. Second, the mean fluorescence intensity within the inner red circle of the top images in Fig. 5d, e, corresponding to the black line in the bottom graphs, which estimates the size of the fusing $\mathrm{SG}$, is much larger, 3.6-fold brighter $\left(\Delta \mathrm{F}_{\text {MassivesG }} / \Delta \mathrm{F}_{\text {SingleSG }}=\right.$ $36,075 / 9,959$ ) for the massive SG fusion (Fig. 5d) compared with single SG fusion events (Fig. 5e). Third, fluorescence intensity between inner and outer red circles (corresponding to grey lines in the bottom graphs) depicting dissipation of SG cargo release is also much larger in the massive SG fusion than that in single SG fusion. We thus considered that these massive fusion events represent genuine compound fusion of several SGs that were already prefused before a lead SG underwent exocytotic fusion with the PM $[8,9,28]$. Two additional examples of compound exocytosis (left 
a

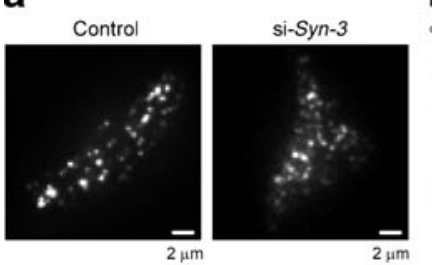

d

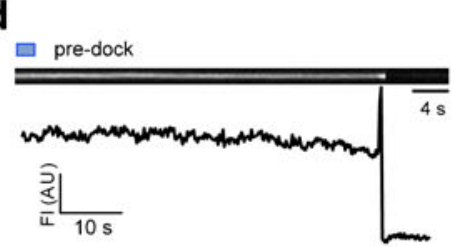

$\square$ newcomer-no dock

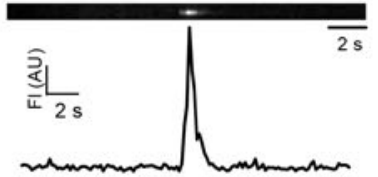

newcomer-short dock

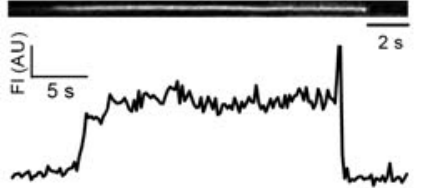

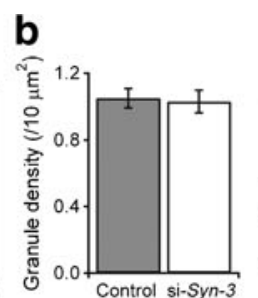

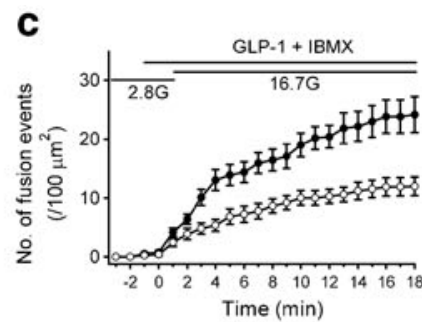

e
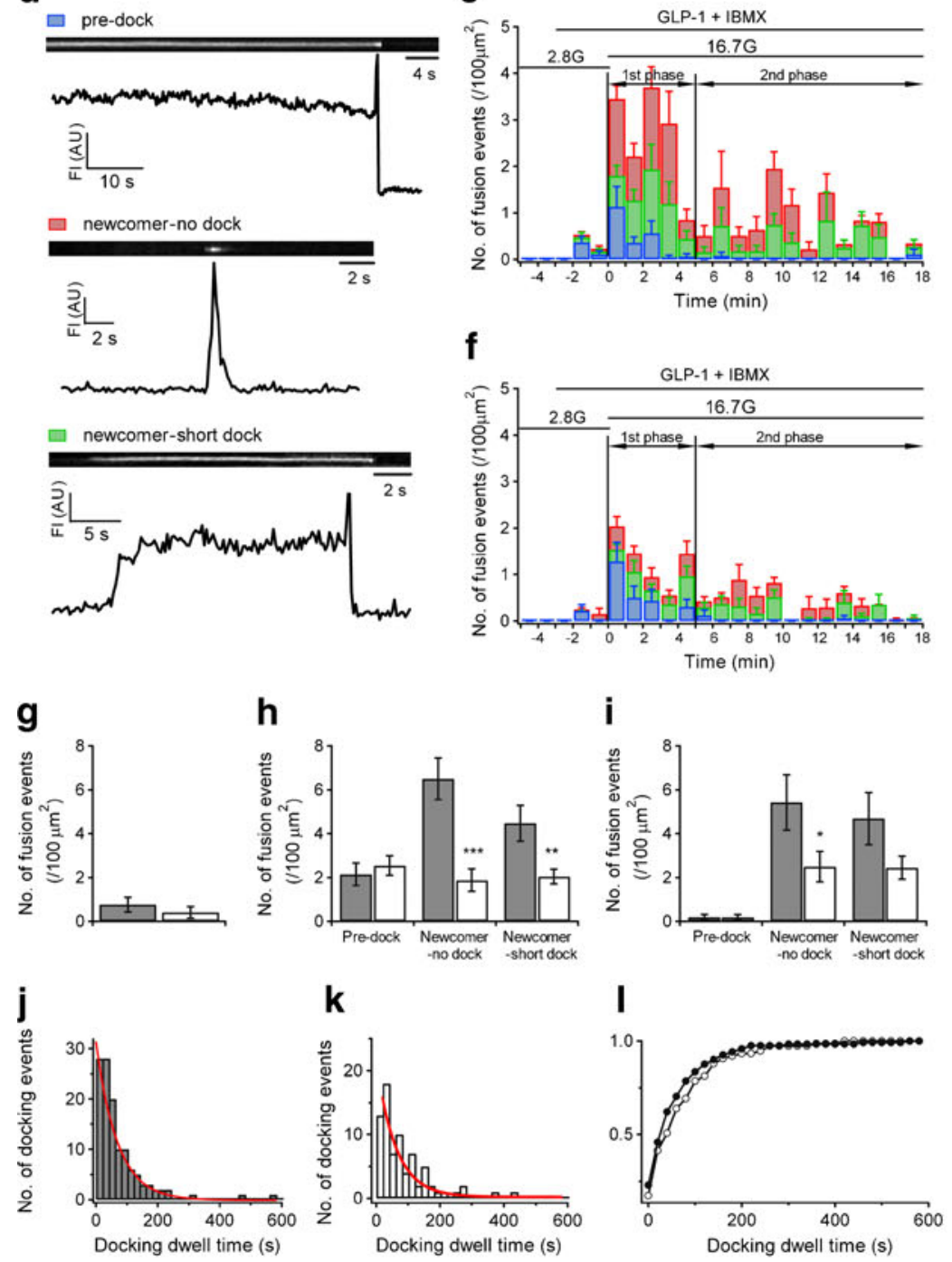

m
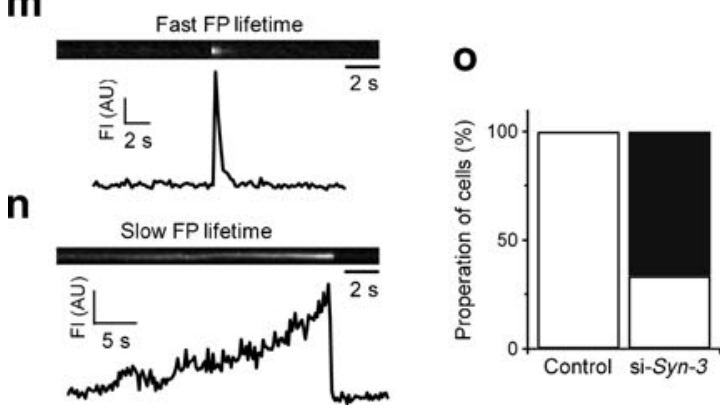

p

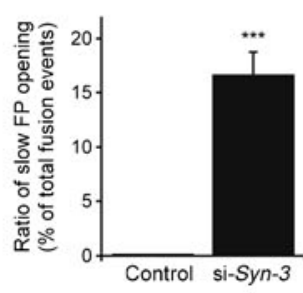


graphs) vs single SG exocytosis (right graphs) are shown in

Fig. 5 g.

We then examined whether loss of function (siRNA knockdown) or gain of function (genetic overexpression) of SYN-3 could influence these SG-SG fusion events in INS-1 cells. Sequential and compound fusion events were assessed and normalised to the footprint area of each cell. Remarkably, SYN-3 knockdown (Fig. 5 h) inhibited $80 \%$ of a

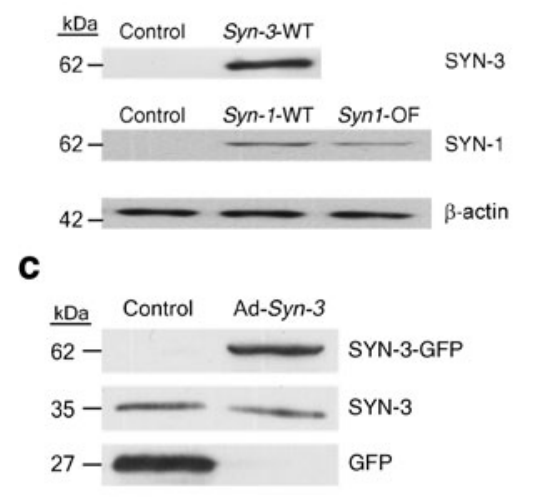

e

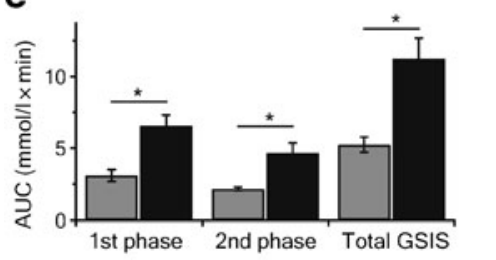

f

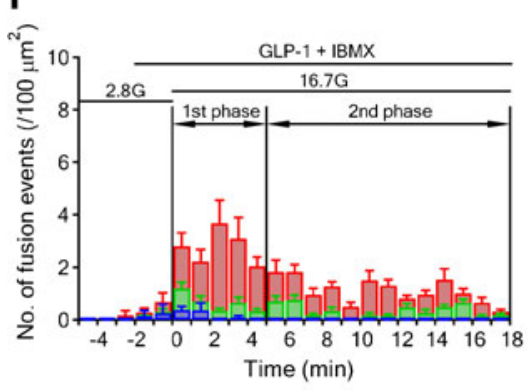

h

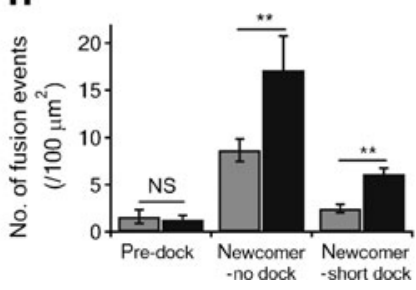

Fig. 4 Increased levels of SYN-3 increase biphasic GSIS by potentiating newcomer granule exocytosis. (a) Western blotting shows SYN-3 levels in INS-1 cells infected with Ad-GFP (control), Ad-Syn-3-GFPWT, Ad-Syn-1-GFP-WT or -OF. (b) Insulin secretion assay from AdSyn-1/-3 overexpressing INS-1 and control cells before (white bars) and after (grey bars) $10 \mathrm{mmol} / 1$ glucose stimulation. Data represent means \pm SEMs of four independent experiments; ${ }^{*} p<0.05$. (c) Western blotting showing levels of SYN-3-GFP (62 kDa), GFP (27 kDa) and endogenous SYN-3 (35 kDa) in mouse pancreatic islets after Ad-Syn3-GFP-WT vs Ad-GFP infection. (d) Islet perifusion assays from mice pancreatic islets infected with Ad-Syn-3-GFP-WT (black circles) and Ad-GFP (control; white circles) stimulated by $16.7 \mathrm{mmol} / 1$ glucose. (e) Corresponding AUC analysis of first phase (11-26 min) and second b

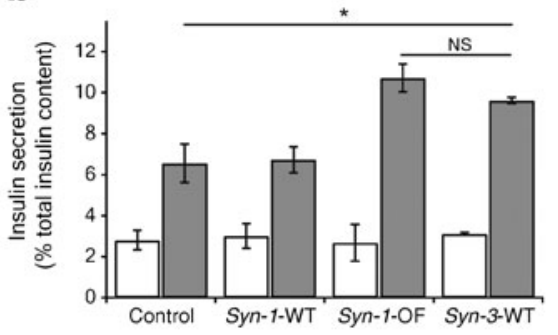

d

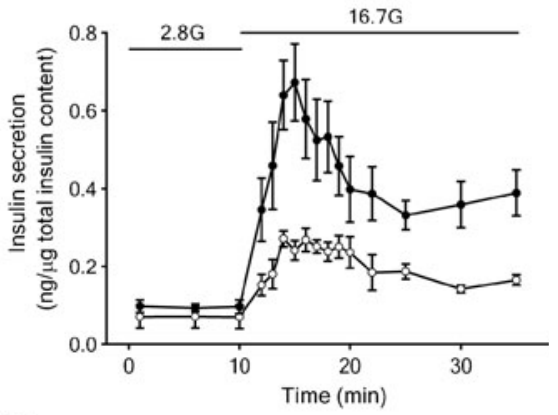

g
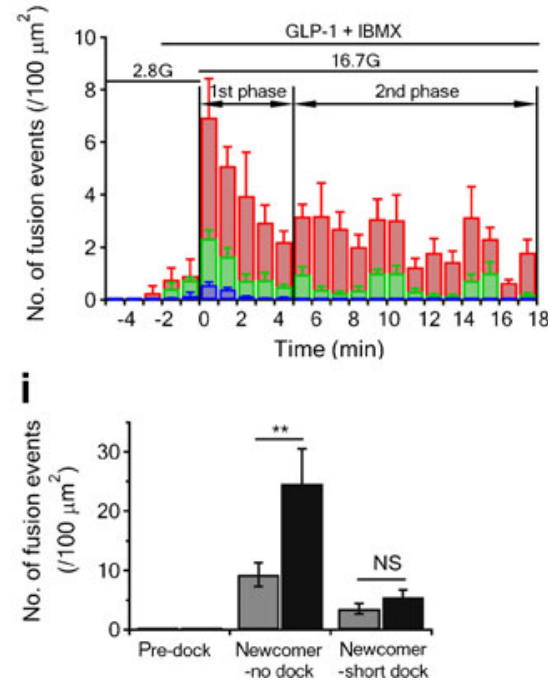

phase (27-40 $\mathrm{min}$ ) GSIS from the perifusion assay in (d). Grey bars, control; black bars, Ad-Syn-3-GFP-WT overexpression. Data represent means \pm SEMs of five independent experiments; ${ }^{*} p<0.05$. (f, g) Histogram of three fusion events under TIRFM in the first phase (first $5 \mathrm{~min}$ after $16.7 \mathrm{mmol} / 1$ glucose stimulation) and second phase (5-18 $\mathrm{min}$ ) in control (f) or Syn-3-overexpressing (g) INS-1 cells. Blue, red and green bars indicate fusion events as described in Fig. 3d. Data were obtained from five independent experiments $(n=2-4$ cells from each experiment), expressed as means \pm SEMs. (h, i) Summary graphs of the three modes of fusion events in first phase (h) and second phase (i), shown as means \pm SEMs. Grey bars, control; black bars, Ad-Syn-3-GFP-WT overexpression. ${ }^{* *} p<0.01$. No., number 
Fig. 5 Increased levels of SYN-3 enhance sequential and compound exocytosis of newcomer SGs. (a) Typical EM images of control and Syn-3 overexpressing islet beta cells after 3 min stimulation with $11.1 \mathrm{mmol} / 1$ glucose and $10 \mathrm{nmol} / 1 \mathrm{GLP}-1$. Scale bars $300 \mathrm{~nm}$. (b) Summary graphs of SG-SG fusion events from mouse pancreatic islet beta cells stimulated as indicated. Summary graphs are expressed as means \pm SEMs. $n=15-23$ per group. Grey bars, control; black bars, AdSyn-3-GFP-WT overexpression. ${ }^{*} p<0.05$. (c) A typical example of sequential exocytosis under TIRFM shown as consecutive images (0.4 s interval) of an SG undergoing exocytosis followed by another SG at the same site (top) with corresponding fluorescence intensity curve (bottom). (d, e) Sequential images of a massive (compound) fusion event (d) and a single SG fusion event (e) under TIRFM, and the corresponding curves of fluorescence intensities from each example. The black and grey curves represent the fluorescence intensities from the inner red circle or from the annulus between the outer and inner red circles drawn in the images above. Note the fluorescence intensity in (d) is threefold greater than that in (e) at the same scale. (f) Twodimensional Gaussian fitting of massive (compound) exocytosis (d) vs that of single SG exocytosis (e). Yellow lines bisecting these images show the positions from where fluorescence-intensity profiles were drawn. These are depicted in the graphs, which show the corresponding fluorescence intensity distribution (grey dotted lines), and Gaussian fitting of this distribution (black lines), from which the FWHM diameters (red arrows) were calculated. (g) Fluorescenceintensity curves from two sets of additional examples of massive (compound) fusion events and single fusion events were drawn at the same scale for each. (h) Percentage of sequential and massive fusion events from control (grey bars) and SYN-3-knockdown (white bars) INS1 cells. (i) Percentage of sequential and massive fusion events from control (grey bars) and Syn-3-overexpressing (black bars) INS-1 cells. $n=15-18$ cells per group. Data were normalised to the unit area of a footprint of each cell under TIRFM, and expressed as means \pm SEMs. ${ }^{*} p<0.05 ;{ }^{* *} p<0.01 .2 .8 \mathrm{G}, 2.8 \mathrm{mmol} / \mathrm{l}$ glucose; $11.1 \mathrm{G}, 11.1 \mathrm{mmol} / \mathrm{l}$ glucose; F, fluorescence; Mas, massive; No., number; Seq, sequential

sequential exocytosis events $\left(0.78 \pm 0.28 / 100 \mu \mathrm{m}^{2}\right)$ when compared with control $\left(4.65 \pm 0.81 / 100 \mu \mathrm{m}^{2}\right)$, but with no significant effect on the number of massive compound fusions. However, overexpressing Syn-3 (Fig. 5i) in INS-3 cells increased not only the sequential but also the multi-granule fusion events by a remarkable twofold and 5.6-fold, respectively.

These results, taken together, lead us to propose that SYN-3 plays a role in regulating SG-SG fusion in beta cells, in addition to mediating the recruitment and exocytosis of newcomer SGs.

\section{Discussion}

In this work, we have demonstrated the novel exocytotic functions of SYN-3, which is present in many cell types but with functions not critically examined. Unlike other SYNs located on the PM, SYN-3 is a t-SNARE on SGs (Fig. 1), appropriately located to serve exocytotic functions different from PM-bound SYNs, most notably SYN-1A, the dominant and putative SYN mediating fusion of docked SGs with the PM $[2,11]$. Here, we showed SYN-3 mediated the recruitment of newcomer SGs to a

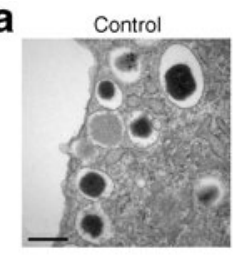

Syn-3 overexpression

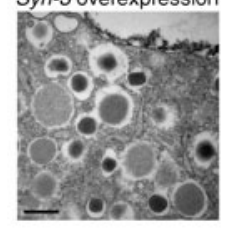

b

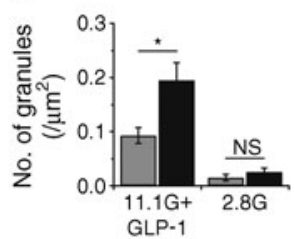

C

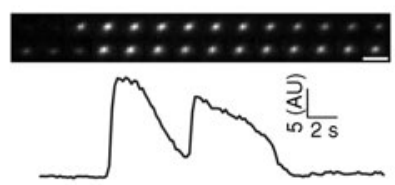

d

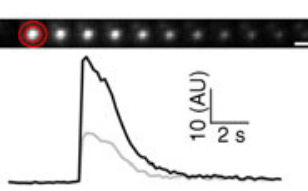

e

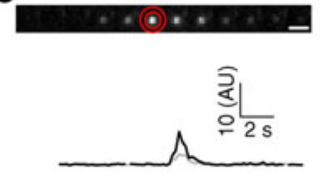

f

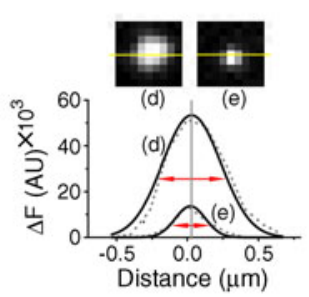

g
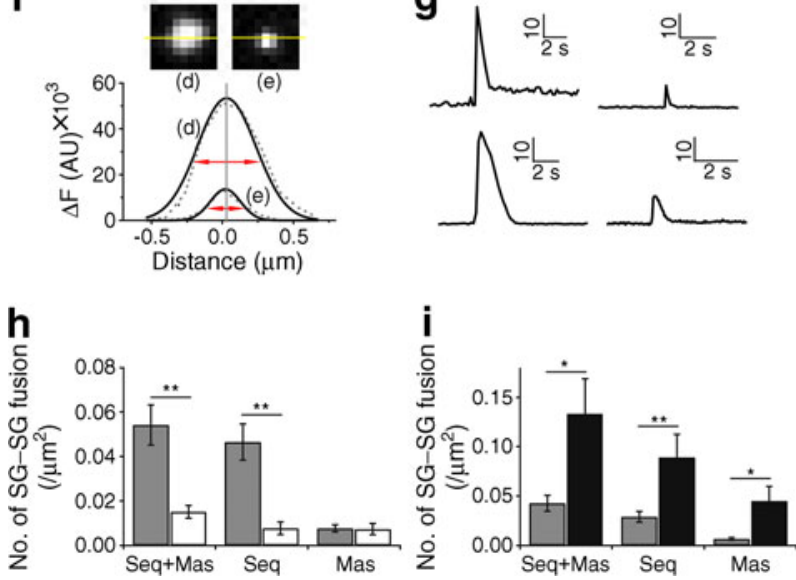

the PM and further induced them to undergo SG-SG fusion, two actions that together greatly amplified the biphasic GSIS.

Neurons have the most efficient primary exocytosis of docked synaptic vesicles, and very rapid and efficient priming machinery to effect further rounds of sustained neurotransmitter release $[2,34]$. Other secretory cells, including neuroendocrine and epithelial cells, have a much lower capacity for primary exocytosis. These secretory cells require alternative exocytotic modes and corresponding fusion machineries to optimise secretion during periods of high physiological demand (i.e. food digestion by pancreatic acinar enzymes) and disease states (i.e. insulin secretion in diabetes, mast cell secretion in allergic reactions) [28]. One mode is fusion of secretory granules, either by orderly sequential fusion as in pancreatic acinar cells $[13,20]$ or by prior homotypic fusion leading to compound exocytosis, as in mast cells [35]. Islet beta cells employ fusion of secretory granules $[31,36]$, but to a much lesser extent than other secretory cells. The other mode is by recruitment of newcomer secretory granules to the PM, first described in 
islet beta cells $[11,37]$, but which could be a mode of exocytosis in other neuroendocrine cells. Here, we show that SYN-3, particularly when produced in abundance to induce gain of function, can enhance both modes of exocytosis on newcomer SGs, without any effect on docked SGs.

It would thus seem that SYN-1A and SYN-3 play nonredundant roles in insulin exocytosis. Interestingly, newcomer SGs, with recruitment mediated by SYN-3, actually accounted for a larger portion of SGs undergoing exocytosis in first-phase GSIS than docked SGs, the latter attributed to SYN-1A [11]. This is of importance, as first-phase GSIS is much reduced in patients with type 2 diabetes, and this has been attributed in part to greatly reduced islet SYN-1A levels [23], along with reduction of cognate SNAREs (SNAP25 and VAMP2) and the Sec1/Munc18-like protein (SM) Munc18a. SYN-3 was shown in pancreatic acinar cells to preferentially form complexes with VAMP8 [20] and Munc18b [38, 39], which are also present in beta cells [40-42]. We recently reported, employing the Vamp8-knockout mice [43], that VAMP8 also mediated the recruitment and fusion of newcomer SGs, and was the putative cognate v-SNARE that binds SYN-3. We showed that Munc13-1 mediated not only the priming of docked SGs [21] but also the newcomer SGs [22], the latter probably by priming SYN-3 exocytotic complexes.

GLP-1 improves GSIS in patients with diabetes [44]; we show here that this may be due, at least in part, to its actions on endogenous SYN-3 on SGs, or SM-SNARE complexes $[29,43,45]$ induced to form with SYN-3. As SYN-3 exocytotic complexes function to enhance the recruitment and fusion of newcomer SGs and SG-SG fusion, such a mechanism may be deployed to compensate for the diabetic defects in docked SGs $[11,46]$ and, if so, this strategy would present itself as a potential therapeutic target [28].

Funding D. Zhu was funded by a postdoctoral fellowship, and E. Koo was funded by a graduate studentship, both from Banting and Best Diabetes Centre (BBDC), University of Toronto.

This work was supported by grants to H. Y. Gaisano (Canadian Institute of Health Research [CIHR] MOP 89889 and MOP 86544).

Duality of interest The authors declare that there is no duality of interest associated with this manuscript.

Contribution statement All the authors contributed to the conception and design of the study, acquisition of data, and analysis and interpretation of data, and drafting of the article and revising it critically for important intellectual content. All authors approved the final version to be published.

\section{References}

1. Rorsman P, Eliasson L, Renstrom E, Gromada J, Barg S, Gopel S (2000) The cell physiology of biphasic insulin secretion. News Physiol Sci 15:72-77
2. Sudhof TC (2004) The synaptic vesicle cycle. Annu Rev Neurosci 27:509-547

3. Kwan EP, Gaisano HY (2009) Rescuing the subprime meltdown in insulin exocytosis in diabetes. Ann NY Acad Sci 1152:154-164

4. Hong W (2005) SNAREs and traffic. Biochim Biophys Acta 1744:493-517

5. Kasai K, Fujita T, Gomi H, Izumi T (2008) Docking is not a prerequisite but a temporal constraint for fusion of secretory granules. Traffic 9:1191-1203

6. Seino S, Takahashi H, Fujimoto W, Shibasaki T (2009) Roles of cAMP signalling in insulin granule exocytosis. Diabetes Obes Metab 11(Suppl 4):180-188

7. Shibasaki T, Takahashi H, Miki T et al (2007) Essential role of Epac2/Rap1 signaling in regulation of insulin granule dynamics by cAMP. Proc Natl Acad Sci USA 104:19333-19338

8. Kwan EP, Gaisano HY (2005) Glucagon-like peptide 1 regulates sequential and compound exocytosis in pancreatic islet beta-cells. Diabetes 54:2734-2743

9. Hoppa MB, Jones E, Karanauskaite J et al (2012) Multivesicular exocytosis in rat pancreatic beta cells. Diabetologia 55:1001-1012

10. Wheeler MB, Sheu L, Ghai M et al (1996) Characterization of SNARE protein expression in beta cell lines and pancreatic islets. Endocrinology 137:1340-1348

11. Ohara-Imaizumi M, Fujiwara T, Nakamichi Y et al (2007) Imaging analysis reveals mechanistic differences between first- and secondphase insulin exocytosis. J Cell Biol 177:695-705

12. Spurlin BA, Thurmond DC (2006) Syntaxin 4 facilitates biphasic glucose-stimulated insulin secretion from pancreatic beta-cells. Mol Endocrinol 20:183-193

13. Pickett JA, Edwardson JM (2006) Compound exocytosis: mechanisms and functional significance. Traffic 7:109-116

14. Lafont F, Verkade P, Galli T, Wimmer C, Louvard D, Simons K (1999) Raft association of SNAP receptors acting in apical trafficking in Madin-Darby canine kidney cells. Proc Natl Acad Sci USA 96:3734-3738

15. Ammar DA, Zhou R, Forte JG, Yao X (2002) Syntaxin 3 is required for cAMP-induced acid secretion: streptolysin Opermeabilized gastric gland model. Am J Physiol Gastrointest Liver Physiol 282:G23-G33

16. Curtis LB, Doneske B, Liu X, Thaller C, McNew JA, Janz R (2008) Syntaxin 3b is a t-SNARE specific for ribbon synapses of the retina. J Comp Neurol 510:550-559

17. Sherry DM, Mitchell R, Standifer KM, du Plessis B (2006) Distribution of plasma membrane-associated syntaxins 1 through 4 indicates distinct trafficking functions in the synaptic layers of the mouse retina. BMC Neurosci 7:54

18. Edwardson JM, An S, Jahn R (1997) The secretory granule protein syncollin binds to syntaxin in a $\mathrm{Ca} 2(+)$-sensitive manner. Cell 90:325-333

19. Gaisano HY, Ghai M, Malkus PN et al (1996) Distinct cellular locations of the syntaxin family of proteins in rat pancreatic acinar cells. Mol Biol Cell 7:2019-2027

20. Cosen-Binker LI, Binker MG, Wang CC, Hong W, Gaisano HY (2008) VAMP8 is the v-SNARE that mediates basolateral exocytosis in a mouse model of alcoholic pancreatitis. J Clin Invest 118:2535-2551

21. Kwan EP, Xie L, Sheu L, Ohtsuka T, Gaisano HY (2007) Interaction between Munc13-1 and RIM is critical for glucagon-like peptide-1 mediated rescue of exocytotic defects in Munc13-1 deficient pancreatic beta-cells. Diabetes 56:2579-2588

22. Xie L, Zhu D, Gaisano HY (2012) Role of mammalian homologue of Caenorhabditis elegans unc-13-1 (Munc13-1) in the recruitment of newcomer insulin granules in both first and second phases of glucose-stimulated insulin secretion in mouse islets. Diabetologia 55:2693-2702

23. Ostenson CG, Gaisano H, Sheu L, Tibell A, Bartfai T (2006) Impaired gene and protein expression of exocytotic soluble N- 
ethylmaleimide attachment protein receptor complex proteins in pancreatic islets of type 2 diabetic patients. Diabetes 55:435-440

24. Yasuda T, Shibasaki T, Minami K et al (2010) Rim2alpha determines docking and priming states in insulin granule exocytosis. Cell Metab 12:117-129

25. Miesenbock G, de Angelis DA, Rothman JE (1998) Visualizing secretion and synaptic transmission with $\mathrm{pH}$-sensitive green fluorescent proteins. Nature 394:192-195

26. Ravier MA, Tsuboi T, Rutter GA (2008) Imaging a target of Ca2+ signalling: dense core granule exocytosis viewed by total internal reflection fluorescence microscopy. Methods 46:233-238

27. Barg S, Olofsson CS, Schriever-Abeln J et al (2002) Delay between fusion pore opening and peptide release from large densecore vesicles in neuroendocrine cells. Neuron 33:287-299

28. Gaisano HY (2012) Deploying insulin granule-granule fusion to rescue deficient insulin secretion in diabetes. Diabetologia 55:877-880

29. Dulubova I, Sugita S, Hill S et al (1999) A conformational switch in syntaxin during exocytosis: role of munc18. EMBO J 18:4372-4382

30. Kishimoto T, Liu TT, Hatakeyama H, Nemoto T, Takahashi N, Kasai H (2005) Sequential compound exocytosis of large densecore vesicles in PC12 cells studied with TEPIQ (two-photon extracellular polar-tracer imaging-based quantification) analysis. J Physiol 568:905-915

31. Takahashi N, Hatakeyama H, Okado H et al (2004) Sequential exocytosis of insulin granules is associated with redistribution of SNAP25. J Cell Biol 165:255-262

32. Fernandez NA, Liang T, Gaisano HY (2011) Live pancreatic acinar imaging of exocytosis using syncollin-pHluorin. Am J Physiol Cell Physiol 300:C1513-C1523

33. Michael DJ, Geng X, Cawley NX et al (2004) Fluorescent cargo proteins in pancreatic beta-cells: design determines secretion kinetics at exocytosis. Biophys J 87:L03-L05

34. Jahn R, Scheller RH (2006) SNAREs - engines for membrane fusion. Nat Rev Mol Cell Biol 7:631-643
35. Alvarez de Toledo G, Fernandez JM (1990) Compound versus multigranular exocytosis in peritoneal mast cells. J Gen Physiol 95:397-409

36. Takahashi N, Kishimoto T, Nemoto T, Kadowaki T, Kasai H (2002) Fusion pore dynamics and insulin granule exocytosis in the pancreatic islet. Science 297:1349-1352

37. Olofsson CS, Gopel SO, Barg S et al (2002) Fast insulin secretion reflects exocytosis of docked granules in mouse pancreatic B cells. Pflugers Arch 444:43-51

38. Kauppi M, Wohlfahrt G, Olkkonen VM (2002) Analysis of the Munc18b-syntaxin binding interface. Use of a mutant Munc18b to dissect the functions of syntaxins 2 and 3. J Biol Chem 277:4397343979

39. Tellam JT, McIntosh S, James DE (1995) Molecular identification of two novel Munc-18 isoforms expressed in non-neuronal tissues. J Biol Chem 270:5857-5863

40. Brunner Y, Coute Y, Iezzi M et al (2007) Proteomics analysis of insulin secretory granules. Mol Cell Proteomics 6:1007-1017

41. Huang X, Sheu L, Kang Y, Eto Y, Kojima I, Gaisano HY (2002) Effects of selective endocrine or exocrine induction of AR42J on SNARE and Munc18 protein expression. Pancreas 25:e56-e63

42. Mandic SA, Skelin M, Johansson JU, Rupnik MS, Berggren PO, Bark C (2011) Munc18-1 and Munc18-2 proteins modulate betacell $\mathrm{Ca} 2+$ sensitivity and kinetics of insulin exocytosis differently. J Biol Chem 286:28026-28040

43. Zhu D, Zhang Y, Lam PP et al (2012) Dual role of VAMP8 in regulating insulin exocytosis and islet beta cell growth. Cell Metab $16: 238-249$

44. Drucker DJ (2006) The biology of incretin hormones. Cell Metab 3:153-165

45. Sudhof TC, Rothman JE (2009) Membrane fusion: grappling with SNARE and SM proteins. Science 323:474-477

46. Kahn BB (1998) Type 2 diabetes: when insulin secretion fails to compensate for insulin resistance. Cell 92:593-596 